\title{
Le choix d'une profession par les étudiants
}

Le cas du Brésil

The Choice of an Occupation by Students - Brazil's Case

La elección de una profesión por los estudiantes - El caso del Brasil

Jean-Jacques Paul et Maria-Luiza Ostheimer-Paul

\section{OpenEdition}

\section{Journals}

Édition électronique

URL : http://journals.openedition.org/ries/1534

DOI : 10.4000/ries. 1534

ISSN : 2261-4265

\section{Éditeur}

Centre international d'études pédagogiques

Édition imprimée

Date de publication : 1 avril 2005

Pagination : 77-86

ISSN : 1254-4590

\section{Référence électronique}

Jean-Jacques Paul et Maria-Luiza Ostheimer-Paul, « Le choix d'une profession par les étudiants », Revue internationale d'éducation de Sèvres [En ligne], 38 | avril 2005, mis en ligne le 21 novembre 2011 consulté le 10 décembre 2020. URL : http://journals.openedition.org/ries/1534 ; DOI : https://doi.org/ $10.4000 /$ ries. 1534 


\section{Le choix \\ d'une profession par les étudiants}

\section{Le cas du Brésil}

\section{Jean-Jacques Paul Maria-Luiza Ostheimer-Paul}

Au Brésil, il n'existe pas de service public d'orientation scolaire (au sens français du terme), comme il est difficile de parler d'un service public de l'éducation. Coexistent, dès le primaire, les écoles régies par les municipalités, les États (le Brésil est un état fédéral) et les écoles privées, rejointes dès le secondaire par des établissements fédéraux, qui voient leur rôle s'affirmer au niveau du supérieur ${ }^{1}$. Cette multiplicité des statuts, associée à la largeur de l'espace occupé par un enseignement privé non subventionné, implique un rôle important de la stratégie des familles pour la carrière scolaire de leurs enfants.

Ce qui pourra étonner un européen, c’est la domination numérique exercée par l'enseignement privé, qui accueille $70 \%$ des étudiants, et qui représente presque neuf institutions sur dix. On remarquera que cette domination, qui a commencé à s'exercer au cours des années 1960, a eu tendance à se renforcer au cours des années récentes. En effet, la part des étudiants inscrits dans les institutions privées est passée de $62 \%$ en 1998 (proportion atteinte au cours des années 1980) à $70 \%$ en 2003. Cet accroissement reflète d'une part la forte demande d'enseignement supérieur parallèlement à une scolarisation plus importante au niveau secondaire, mais également la plus grande réactivité du secteur privé à cette demande.

Nombre d'institutions d'enseignement supérieur et étudiants inscrits, par catégorie administrative - Brésil - 1998-2003

\begin{tabular}{|l|c|c|c|c|c|c|}
\hline & \multicolumn{3}{|c|}{ Institutions } & \multicolumn{3}{c|}{ Étudiants inscrits } \\
\hline Année & Total & Publiques & Privées & Total & $\begin{array}{c}\text { Inst. } \\
\text { publiques }\end{array}$ & $\begin{array}{c}\text { Inst. } \\
\text { privées }\end{array}$ \\
\hline 1998 & 973 & 209 & 764 & 2125958 & 804729 & 1321229 \\
\hline 1999 & 1097 & 192 & 905 & 2369945 & 832022 & 1537923 \\
\hline 2000 & 1180 & 176 & 1004 & 2694245 & 887026 & 1807219 \\
\hline 2001 & 1391 & 183 & 1208 & 3030754 & 939225 & 2091529 \\
\hline 2002 & 1637 & 195 & 1442 & 3479913 & 1051655 & 2428258 \\
\hline 2003 & 1859 & 207 & 1652 & 3887771 & 1137119 & 2750652 \\
\hline
\end{tabular}

Source: Inep/MEC.

1. Voir sur l'organisation du système éducatif brésilien l'article de Cécilia Regina Vendramini pp. 131-137. 
Après avoir présenté les modalités d'accès à l'enseignement supérieur, nous mettrons en évidence les enjeux du choix d'études avant d'évoquer dans un dernier paragraphe les problèmes liés à l'information sur les carrières professionnelles.

\section{L'ACCÈs À L'ENSEIGNEMENT SUPÉRIEUR}

L'entrée dans l'enseignement supérieur brésilien s'effectue sur la base d'un concours, nommé Vestibular. Ce dispositif est mis en œuvre par toutes les universités publiques et la grande majorité des universités privées brésiliennes. Chaque université est autonome dans l'élaboration et l'organisation du concours. Parfois, plusieurs établissements se regroupent autour d'un concours unique mais cette forme est minoritaire. Fréquemment, il existe deux phases : une phase éliminatoire et une phase classificatoire. Les candidats doivent choisir avant le concours leur filière de prédilection et les épreuves comprennent à la fois une partie commune et une partie spécifique à une filière ou un groupe de filières. Les candidats passent en général le concours de plusieurs établissements d'enseignement supérieur, car le résultat pour chacun d'entre eux est l'admission ou l'ajournement. Les chances de réussite dépendent bien évidemment du rapport entre le nombre de candidats et le nombre de places.

En 1998, le ministère de l'Éducation a créé l'ENEM - l'Examen national de l'enseignement moyen. Il s'agit d'une procédure d'évaluation de la performance de l'élève à la fin de l'enseignement secondaire, qui fonctionne comme modalité alternative ou complémentaire du processus de sélection dans les différents secteurs du marché du travail et ainsi que pour les examens d'admission aux filières de formations professionnelles courtes (cours techniques supérieurs) et l'enseignement supérieur.

Actuellement, plus de quatre cents universités et facultés dans tout le pays utilisent l'ENEM différemment, du fait de leur autonomie, pour définir leurs critères de sélection : certaines dispensent les candidats du Vestibular (concours d'admission), d'autres, par exemple, intègrent les notes de l'ENEM dans leurs critères de sélection.

C'est véritablement à partir de 2001 que l'ENEM va se développer, avec l'instauration de sa gratuité. Cette année-là, $51 \%$ des élèves qui avaient achevé l'enseignement secondaire ont passé l'ENEM ; ils n'étaient encore que $14 \%$ en 2000 (Cortelazzo, 2003). En 2001, un peu plus des deux tiers des candidats avaient achevé l'enseignement secondaire au cours de cette même année, alors qu'un tiers l'avait conclu antérieurement.

Cortelazzo (2003) fournit l'exemple des deux universités prestigieuses de l'État de São Paulo que sont l'USP et l'UNICAMP. Elles prennent en compte les notes de l'ENEM pour un cinquième de la note de la première phase du 
Taux de réussite au Vestibular 2000 en fonction du passage de l'ENEM

\begin{tabular}{|l|l|c|c|}
\hline & & Sans ENEM & Avec ENEM \\
\hline \multirow{2}{*}{ UNICAMP } & Privé & $5,6 \%$ & $6,4 \%$ \\
\cline { 2 - 4 } & Public & $4,0 \%$ & $7,7 \%$ \\
\hline \multirow{2}{*}{ USP } & Privé & $4,4 \%$ & $8,5 \%$ \\
\cline { 2 - 4 } & Public & $1,8 \%$ & $5,2 \%$ \\
\hline
\end{tabular}

concours (les autres quatre cinquièmes provenant de la note aux épreuves du concours). Les données analysées proviennent de l'ENEM de 1999 et du Vestibular de 2000. Elles montrent que l'ENEM a été passé par une proportion plus importante d'élèves issus des établissements privés ( $88 \%$ des étudiants issus du privé dans le cas de l'UNICAMP) que d'établissements publics (64\% des étudiants issus du pubic dans le cas de l'UNICAMP). Ce résultat peut provenir du fait qu'à l'époque l'examen était payant et que les élèves des écoles privées sont d'origine sociale plus favorisées que ceux des écoles publiques.

D'après ces résultats obtenus à partir des données de Cortelazzo (2003), il apparaît que le taux de réussite au Vestibular des anciens élèves des lycées publics reste inférieur à celui des anciens élèves du privé dans le cas de l'USP (ce qui renvoie à la fois à des caractéristiques d'élèves et d'établissements scolaires), mais que la prise en compte des résultats à l'ENEM plus avantageuse pour les élèves des écoles publiques conduit à les doter d'un taux de réussite plus élevé à l'UNICAMP. La réforme de l'ENEM aurait donc eu dans ce cas un effet égalisateur.

L'enquête du Ministère de l'Éducation révèle que pour la première fois à la rentrée 2003 le nombre de places offertes par les institutions d'enseignement supérieur a été supérieur au nombre d'élèves qui ont achevé le cycle de l'enseignement moyen (deux millions de places à rapporter à 1,9 million d'élèves). Une proportion importante de places est restée vacante dans les institutions privées $(42,2 \%)$ mais ce phénomène n’a pas épargné les institutions publiques puisque le taux de vacance s'est élevé à $5,1 \%$. Alors que ce taux est resté identique pour les institutions publiques entre 2002 et 2003, il a augmenté de cinq points de pourcentage pour les institutions privées, ce qui montre le dynamisme de ce secteur.

\section{LES ENJEUX DU CHOIX D’ÉTUdES}

Toutes les filières de l'enseignement supérieur ne sont pas recherchées avec la même intensité par les candidats. Par exemple, lors du Vestibular de 1993 à l'UFRJ (Université Fédérale de Rio de Janeiro) (Paul et Do Valle, 1998), le rapport était compris entre vingt-huit candidats par place en médecine, vingt-six en odontologie, quinze en communication sociale, douze en droit et 
des valeurs bien plus basses comme deux en lettres et sciences sociales et moins de un pour des filières comme arts de la scène et musique.

Afin d'augmenter leurs chances au Vestibular, il est fréquent que les candidats s'inscrivent préalablement dans des institutions spécialisées par la préparation à ce concours : les cursinhos ou pré-vestibular. De nombreuses écoles privées incluent cette préparation dans la troisième année d'études secondaires moyennes. Dans l'étude de Moretto (2004), $53 \%$ des étudiants de l'université publique ont fréquenté le pré-vestibular pendant un an et $27 \%$ pendant plus d'un an. Les proportions respectives pour les étudiants des institutions privées sont de $38 \%$ et $11 \%$, ce qui montre la plus grande difficulté à intégrer l'université publique (l'USP en l'occurrence dans cette étude).

Une spécificité importante du système universitaire public brésilien est sa gratuité ; mais il n'existe aucun système de bourse destiné à permettre aux étudiants de faire face à leurs frais d'intendance. Un système de prêt existe épisodiquement, qui sert à financer partiellement les droits d'inscription dans les universités privées. En septembre 2004, l'actuel gouvernement a lancé le "programme Université pour Tous», destiné à offrir des places d'études gratuites dans les universités privées à des étudiants pauvres, avec une attention particulière portée également en faveur des étudiants descendants d'africains ou d'indiens. Il s'agit d'un système de bourses destinées au paiement des droits. En fonction du revenu des parents, il peut s'agir de bourses à taux plein ou à taux de $50 \%$. Le financement doit être assuré par les propres établissements d'enseignement privé, en échange de réductions d'impôts. L'objectif annoncé est de pouvoir accueillir dans ces conditions 300000 étudiants pauvres dans les cinq années.

Les données sur les droits d'inscription sont rares. Paul et Wolff (1996) montrent à partir de quelques études de cas que les montants de droits d'inscription pouvaient considérablement varier. $\mathrm{Si}$ on divise les filières d'études en quatre catégories (sciences sociales et gestion; sciences de l'ingénieur; médecine; sciences humaines et formation de maîtres), les droits d'inscription exigés en 1989 dans huit types d'institutions privées différents se montraient les plus élevés dans le secteur de la santé, comme on pouvait s'y attendre, en allant de 1500 à 3500 dollars américains annuels (payables en douze mois). Les écoles d'ingénieurs les suivaient avec 1500 à 2400 dollars américains et les droits les moins élevés correspondaient aux filières des sciences humaines et sociales (de 600 à 1800 dollars américains). En général, les institutions municipales et les facultés religieuses demandaient les sommes les plus faibles, tandis que les plus élevées étaient réclamées par les universités privées.

Paul et Wolff (1996) montrent la continuité qui existe entre les différents types d'institutions quand on se réfère aux filières choisies par les étudiants. Ainsi, le statut socio-économique des étudiants apparaît varier sensiblement selon la filière. Les institutions privées sont hétérogènes et offrent une 
diversité de filières à des étudiants ayant des origines socio-économiques variées. Les filières les plus prestigieuses, comme médecine et sciences de l'ingénieur, attirent plus fréquemment les étudiants d'origine sociale favorisée. L'élément essentiel du panorama est que tous les étudiants préfèrent les établissements fédéraux parce qu'ils sont gratuits et prestigieux. Si un étudiant favorisé n'est pas admis dans une filière prestigieuse d'une université fédérale, alors il se rabattra sur la même filière dans une institution privée. Un étudiant de statut socio-économique plus modeste choisit en général des filières moins prestigieuses soit dans des institutions publiques ou privées. Il faut relever que les universités fédérales offrent moins de cours en soirée, ce qui rend leur fréquentation difficile pour des étudiants qui doivent travailler la journée.

L'existence d'un concours d'entrée ne garantit pas que la majeure partie des admis dans les universités terminent leurs études. Les abandons sont fréquents, et certains, devant le taux d'évasion élevé et les fréquentes migrations d'une filière à l'autre dans les universités, recommandent l'instauration de services d'orientation et d'information au sein des universités, capables d'informer les candidats sur le contenu des cours, les professions préparées et leurs chances de réussite eu égard à leurs caractéristiques (Bandeira Andriola, 2003).

Paul et Wolff (1996) mettent en évidence l'importance des taux d'abandon des différentes filières. D’après leurs estimations, environ un étudiant sur deux seulement mène à son terme les études entreprises. En ce qui concerne les filières d'études, la santé présente les taux de d'achèvement les plus élevés, en confirmant les hypothèses que les filières exigeant une haute sélectivité et promettant des salaires élevés sont plus susceptibles de retenir les étudiants. Toutefois, une filière moyennement rémunérée comme les sciences agraires, retient également les étudiants, tandis que les études d'ingénieur, que l'on pouvait espérer présenter un taux élevé d'achèvement, ne fait pas mieux que lettres et sciences sociales. Cela peut être le résultat d'un niveau d'exigence académique excessivement élevé de la part des enseignants, provoquant ainsi des départs des études d'ingénieurs vers des filières moins exigeantes.

Le temps mis pour achever ses études apparaît également différer selon le statut de l'institution d'enseignement. Alors que les étudiants mettaient en moyenne 4,5 années pour obtenir leur diplôme de graduation (équivalent à la maîtrise française), cette durée s'élève à 6,1 années pour les universités fédérales mais s'abaisse à quatre années pour les facultés privées. Ces différences peuvent être dues au fait que le paiement de droits d'inscription dans les établissements privés conduit les étudiants à ne pas retarder leur progression académique; on ne peut aussi exclure l'hypothèse que les établissements privés sont moins exigeants en termes académiques. Cependant, les modèles de gains établis par les auteurs ne montrent pas de différences de salaires entre diplômés des universités fédérales et privées. 
Les résultats montrent également qu'odontologie/médecine ont les salaires les plus élevés, suivis par informatique, comptabilité, gestion/économie/droit, sciences de l'ingénieur, chimie/statistique, arts ménagers/ services bibliothécaires, services auxiliaires médicaux, nutrition/services sociaux, agronomie, et formation de maîtres. Les femmes gagnent d'une manière frappante moins que les hommes $(-60,8 \%)$, même après avoir pris en compte la filière. S'affirme également une influence de l'origine sociale: les diplômés ayant des parents avec niveau de scolarité plus élevé gagnent davantage.

\section{LA FAIBLESSE DE L'INFORMATION SUR LES CARRIÈRES PROFESSIONNELLES}

La complexité concerne également les carrières que les étudiants peuvent préparer et à propos desquelles les informations sont réduites.

La revue Veja dénombre 570 professions, qui peuvent se ramifier en un nombre supérieur de carrières. Ces professions peuvent être préparées dans des filières multiples, au sein d'institutions diverses, qui exigent des montants de droits variés, avec des chances de réussite au concours d'entrée différentes selon les filières et les institutions.

Le Guide de l'Étudiant a recensé dix-sept institutions d'orientation professionnelle, en général localisées dans les départements de psychologie d'universités, publiques et privées, qui accueillent gratuitement (pour douze d'entre elles) ou pour un prix modeste (pour cinq d'entre elles, autour de 8 euros) les jeunes à la recherche d'informations sur les carrières et sur leurs propres aspirations et possibilités. En général, les services articulent entretiens individuels et réunions en groupes de quinze à vingt personnes, au cours desquelles sont présentées des informations sur les professions et les carrières et sont mises en œuvre des techniques de connaissance de soi. On pourra rapporter cet effectif de dix-sept institutions qui offrent des services d'orientation professionnelle avec le nombre total de 1859 institutions recensées.

Il existe également des orienteurs travaillant pour leur propre compte. La revue Veja (2004) en a dénombré environ 298 dans les listes officielles répartis sur 19 états (sur 27).

La recherche menée en 2001 par Moretto (2004) auprès d'un échantillon de 2131 étudiants de première année de cinq universités de la municipalité de São Paulo (une publique et quatre privées) semble indiquer que les étudiants, malgré la complexité du système et l'inorganisation du processus d'orientation professionnelle, conduisent une stratégie dont les résultats les satisfont.

En ce qui concerne le moment du choix de la filière, un tiers des étudiants l'a effectué au cours de la dernière année de l'enseignement moyen et 
$28 \%$ à la veille même du Vestibular. À l'opposé, 39\% ont fait leur choix dès l'enseignement de base ou au cours de la première année de l'enseignement moyen. Les choix précoces sont caractéristiques des étudiants qui se trouvent dans les filières les plus demandées (la proportion de ceux qui ont choisi leur filière dès l'enseignement de base est de respectivement de $56 \%$ pour médecine et $44 \%$ pour odontologie). À l'inverse, $53 \%$ des étudiants en lettres des institutions privées ont choisi la veille du Vestibular.

$\mathrm{Au}$ moment du choix, $52 \%$ des étudiants étaient décidés pour une seule filière, les autres hésitaient avec une filière voisine $(28 \%)$ ou avec une filière complètement différente $(20 \%)$. Finalement, $79 \%$ déclarent se trouver dans la filière qu'ils ont choisie $(90 \%$ avec ceux qui sont dans une filière voisine). Ils considèrent que leur niveau d'information est correct, puisque $69 \%$ d'entre eux se déclarent partiellement informés et $27 \%$ totalement informés. Les sources d'informations sont à $30 \%$ les professionnels du domaine, à $24 \%$ les amis et les proches, $21 \%$ les journaux, guides et revues reliés au Vestibular, $24 \%$ les revues spécialisées dans le domaine. Pour ce qui a trait aux raisons du choix, $88 \%$ estiment s'être décidés en fonction de leur volonté de réalisation personnelle, de leurs aptitudes et leur vocation pour la filière et la profession; $78 \%$ ont tenu compte de la qualité et du prestige de l'institution, $73 \%$ des opportunités sur le marché du travail, $65 \%$ des possibilités d'ascension financière et $44 \%$ des attentes des parents.

Finalement, $60 \%$ des étudiants suivent leur première option et estiment avoir fait le meilleur choix. Les auteurs relèvent cependant qu'un tel résultat est à rapprocher du fait que l'enquête porte sur des étudiants qui ont réussi à intégrer une université et que, par ailleurs, un phénomène de rationalisation a posteriori n'est pas à exclure. En outre, les données présentées par Paul et Wolff (1996) insistent sur l'importance de l'abandon au cours des études.

L'économiste du travail Roberto Macedo a récemment publié un livre où il traite de la question du choix de la profession et de la filière d'étude par les adolescents et qui s'intitule "Son diplôme, sa planche». Il utilise ainsi la parabole du surfeur pour décrire le candidat au Vestibular à la recherche d'un bon diplôme ou d'une bonne planche. Il la prolonge pour décrire le bon surfeur qui va choisir la bonne vague, comme le diplômé qui ne va pas hésiter à évoluer en fonction des opportunités du marché du travail.

Macedo invite à multiplier les sources d'informations, à chercher à mieux se connaître, à développer son potentiel, à collecter des informations sur les professions, les carrières, les filières, à compléter les études suivies par des cours supplémentaires, en langues ou en informatique. Selon Macedo, il serait préférable d'instaurer dans les universités un cycle de base de deux ans, laissant le choix de la profession ou de la filière professionnelle à l'issue de ce cycle. Cet ouvrage illustre le caractère individuel du choix professionnel au Brésil. 
Dans un rapport préparé pour le Ministère du Travail brésilien, Macedo (2000) regrette l'habitude brésilienne de raisonner en termes de métier rattaché à la formation et de ne pas tenir assez compte de l'éventail des professions exercées à l'issue de chacune des filières de formation. Selon lui, cette habitude est entretenue par l'absence de données sur le fonctionnement concret du marché du travail, et en particulier sur les professions effectivement exercées par les diplômés. Il appelle à la réalisation d'enquêtes systématiques, dont les résultats pourraient alimenter les documents utilisés pour l'information et l'orientation professionnelle.

Il faut préciser que ces dernières années, les pouvoirs publics ont visé à standardiser la qualité des différentes filières de l'enseignement supérieur, par un système d'évaluation complexe, qui s'appuie notamment sur une pratique originale d'évaluation des acquisitions des étudiants pour évaluer les filières des différentes institutions. À la fin de l'année académique 2004, 2187 «cours» devaient être évalués, représentant 13 domaines d'étude et impliquant 156000 étudiants (96000 entrants et 60000 en fin de formation). Le ministère prévoit que les résultats des évaluations soient mis à la disposition des parents et des étudiants pour faciliter les choix d'études et d'établissements.

Les données qui précèdent montrent que les problèmes relatifs à l'orientation dans l'enseignement supérieur brésilien sont à la fois spécifiques et communément partagés. Leur spécificité repose essentiellement sur le caractère libéral de l'organisation universitaire. Les universités présentent des statuts diversifiés qui tiennent à la fois à leur niveau de décentralisation et/ou à leur caractère privé ou public. Les prix d'acquisition de la formation sont très fortement dépendants du caractère de l'université à laquelle on s'adresse et au sein de celle-ci, de la filière choisie; les stratégies de scolarisation sont fortement individualisées. L'enseignement universitaire brésilien semble donc présenter bon nombre des caractéristiques d'un marché, sans doute imparfait en termes économiques dans la mesure où la concurrence et l'information n'y sont pas parfaites.

Les caractères les plus communément partagés avec les autres pays du monde reposent d'une part sur la structuration des formations, et d'autre part sur la sociologie des formés. Les universités et, au sein de celles-ci, les filières sont structurées sur une hiérarchie essentiellement fondée sur les perspectives d'emploi et de gains qu'elles promettent. Les filières médicales et d'ingénieurs occupent les premières places tant au niveau des coûts que de l'accueil des meilleurs étudiants. Les conséquences de cette structuration sont fortes sur le plan sociologique : les filières les plus huppées et les plus prometteuses d'avenir sont aussi celles qui sont fréquentées par les publics d'étudiants issus des 
milieux les plus favorisés. Mais en cela le Brésil diffère-t-il si sensiblement de pays à régulation étatique plus forte comme la France?

Ces caractéristiques et l'absence d'un service public d'information et d'orientation alourdissent le caractère pénalisant de l'origine sociale des étudiants les plus défavorisés. Un service public d'information et d'orientation a, parmi ses fonctions, d'égaliser les différences d'accès à l'information sur les études et les professions en permettant à ceux dont les parents n'ont pas connu la scolarisation choisie d'accéder à des données objectives leur permettant d'étayer leurs choix. L'absence de ces services ne permet qu'aux parents bien informés d'informer leurs enfants et contraint les autres à payer un service privé d'information lorsqu'ils le peuvent.

Dans la mesure où ils ne reçoivent pas de conseil en matière d'orientation scolaire et professionnelle dans leurs écoles, les jeunes cherchent à combler leur manque d'information en se documentant à travers les médias, en faisant appel à des cabinets d'orientation, en recourant à des tests... Pourtant, les informations existent sur le prestige des institutions, les opportunités sur le marché du travail, et les possibilités d'ascension sociale et financière. Mais pour en disposer encore faut-il que l'étudiant bénéficie d'un environnement qui lui permette d'y accéder et de les mobiliser.

\section{BIBLIOGRAPHIE}

BANDEIRA ANDRIOLA W. (2003): «Evasão discente na Universidade Federal do Ceará : proposta para identificar causas e implantar um Serviço de Orientação e Informação", in Ensaio: Avaliação e Políticas Públicas em Educação, $\mathrm{n}^{\circ}$ 40, vol. 11, juillet-septembre, pp. 332-347.

CORTELAZZO A.L. (2003): "Utilização do ENEM pelas Universidades Estaduais Paulistas: Abordagem quantitativa da abrangência do exame e desempenho dos egressos de escolas públicas e privadas de ensino médio ", in Ensaio: Avaliação e Políticas Públicas em Educação, n 39, vol. 11, avriljuin, pp. 210-221.

Guia Abril do Estudante 2000 (1999): São Paulo: Editora Abril.

GUIMARÃES DE CASTRO M.H. (2005): «O Pisa mostrou: educação no Brasil melhora», in O Estado de São Paulo, $1^{\mathrm{er}}$ janvier.

MACEDO R. (1998): Seu diploma, sua prancha. Como escolher a profissão e surfar no mercado de trabalho. Editora Saraiva.

MORETTO Cl.F. (2004): «Os processos de decisão no ensino superior. Algumas evidências para os universitários do município de São Paulo» in Revista Econômica Contemporânea. Rio de Janeiro: 8 (1), janvier/juin, pp. 183-209. 
PAUL J.-J., DO VALLE SILVA N. (1998) : "Conhecendo o seu lugar: A autoseleção na escolha de carreira» in Revista Brasileira de Política e Administração da Educação. Brasília, vol.4, nº 1, janvier/juin, pp. 115-130.

PAUL J.-J., WOLFF L. (1996): «The Economics of Higher Education in Brazil» in Opportunity Foregone: Education in Brazil, sous la direction de Nancy Birdsall et Richard H. Sabot. Washington: John Hopkins Univ. Press, pp. 523-554.

Revue Veja (2004): Sao Paulo: Editora Abril, juin.

WERTHEIN J. (1998): "A vez do ensino médio», in Folha de São Paulo, septembre. 